\section{Barreras y acciones para mejorar la calidad y el impacto de Acta Pediátrica de México}

\author{
Barriers and actions to improve \\ the quality and impact of Acta \\ Pediátrica de México
}

Acta Pediátrica de México se publica desde 1980 de manera puntual e ininterrumpida; es el Órgano Oficial del Instituto Nacional de Pediatría. Es una publicación bimestral que tiene como propósito fundamental la difusión de evidencia científica y de información generada como producto de investigación original básica, clínica, epidemiológica y social en el campo de la pediatría, que permita generar y mejorar los modelos de atención a la salud durante la infancia y la adolescencia. Desde 2013, el equipo editorial de la revista se renovó y desde entonces hemos identificado barreras e implementado acciones para lograr mejorar su calidad e impacto.

Proceso de sometimiento para publicación

Actualmente, nuestra revista recibe propuestas de manuscritos para su publicación a través de la plataforma Open Journal Systems (OJS, www.ojs.actapediatrica.org.mx), con lo cual se pretende garantizar que el autor que envía el manuscrito haya leído, comprendido y aceptado nuestras normas de autor y políticas de gestión editorial. Una primera revisión se asegura que el manuscrito esté en un formato digital apropiado y estructura acorde al tipo de publicación (artículo original, de revisión, caso clínico de interés especial o de sesión anatomo-clínica del Instituto Nacional de Pediatría, criterio pediátri$\mathrm{co}$, editorial). Para mantener anonimato durante el proceso de revisión por pares, se requiere que el manuscrito no contenga datos que permitan identificar el origen de la publicación (e.g. nombres de instituciones, nombres de autores, etc).

Desafortunadamente, en muchas ocasiones, la clasificación de los manuscritos no coincide con el tipo de publicación real; las estructuras no cumplen con lo solicitado en nuestras normas de autor; contienen datos que permiten identificar el origen de la publicación; los documentos anexos se envían en formatos inapropiados, no se envían cartas de aprobación o copia del consentimiento o asentimiento informado cuando es necesario. En un primer filtro, estos manuscritos los rechazamos y regresamos a los autores especificando las razones; éstos, pueden corregir, completar, modificar sus manuscritos y volverlos a enviar. Lo anterior, consume tiempo y trabajo innecesario; por tal motivo, hemos incorporado una lista de cotejo como documento anexo obligatorio para el envío de un manuscrito. Esta lista tiene todos los requisitos mínimos indispensables y el autor 
debe especificar en qué página de su manuscrito se encuentra. Las listas de cotejo son específicas para cada tipo de publicación y están basadas en lineamientos de aceptación internacional disponibles en (www.equator-network.org). De esta forma, los autores podrán identificar omisiones y corregirlas antes de enviar el manuscrito, asimismo se estandariza la estructura de los manuscritos en base a recomendaciones consensuadas internacionales.

\section{Asignación de revisores y comité editorial}

Desde 2013, Acta Pediátrica de México ha reestructurado, diversificado y enriquecido su comité editorial, mediante la invitación de colaboradores de otras instituciones y países. Actualmente, nuestro comité cuenta con 42 elementos fijos, de los cuales (20) 48\% son de otra institución; otro $20 \%$ son extranjeros de los cuales el $100 \%$ pertenecen a algún órgano institucional de investigación de sus respectivos países y el $50 \%$ de nuestro comité editorial nacional pertenece al Sistema Nacional de Investigadores.

Cuando el manuscrito cumple con lo antes descrito, lo enviamos a un editor de sección quien identifica la especialidad o área de conocimiento a la que pertenece y selecciona dos revisores de nuestro comité de arbitraje y un formulario de revisión. Este último, consiste en la misma lista de cotejo que se le solicita al autor al enviar su manuscrito, sumado a una evaluación de la validez de la información que pretende publicarse; una sección abierta para comentarios específicos con respecto al contenido y redacción del manuscrito $y$, finalmente, un apartado de dictamen (i.e. aceptado sin cambios, aceptado con cambios menores, aceptado con cambios mayores, rechazado).

Uno de los principales puntos a mejorar, y que impacta de manera muy negativa la gestión editorial, es que el tiempo de respuesta de nuestros dictaminadores continúa siendo mayor al que planteamos como objetivo. La media de tiempo para dictamen es de 4 semanas, ( 1 a 15 semanas). Por otro lado, la proporción de revisores de nuestro comité sobre-representa a nuestra institución.

Para tratar de disminuir el tiempo de respuesta al autor que publica en Acta Pediátrica de México, la invitación a los revisores cuenta con una solicitud expresa de realizar el proceso antes de una fecha límite, mandamos recordatorios a nuestros revisores a través de la plataforma OJS cuando se acerca la fecha de entrega (5 días antes), y en caso de pasar la fecha límite, enviamos recordatorios 2 veces por semana.

Nuestro comité editorial está en constante evaluación y reestructuración, nuestro objetivo es incrementar el número de revisores de otras instituciones, nacionales e internacionales, maximizar la proporción de miembros pertenecientes a sistemas institucionales de investigación, y minimizar el número de revisores que no participan activamente o satisfactoriamente con el proceso de revisión. La diversificación, profesionalización y depuración del comité de arbitraje permitirá tener revisiones de mejor calidad y en tiempos más oportunos.

\section{Dictamen, maquetación y publicación}

El $40 \%$ de los manuscritos que son sometidos a nuestra revista son rechazados en un primer evento. Del $60 \%$ restante, el $100 \%$ requiere al menos de correcciones menores, por lo que se regresa a los autores (con el consiguiente retraso en publicación) para que revisen los comentarios, correcciones y sugerencias que los evaluadores hayan expresado. Una vez corregido el manuscrito, se asignan a dos especialistas que harán comentarios y sugerencias a los autores, los cuales enviarán versiones subsecuentes que pasarán por el mismo proceso hasta obtener 
un dictamen de aceptado para publicación o rechazado.

Los manuscritos que son aceptados, se envían a la editorial para realizar los cambios de formato y generar la versión final de los artículos y nos envían "pruebas de diagramación/maquetación" para revisión final. Tras la revisión de éstas, se solicitan correcciones finales y se publica la versión final de los manuscritos en formato PDF. Subimos el material a través de la plataforma OJS para publicar el número correspondiente, durante este proceso se genera y asigna un número DOI para cada artículo, mismos que se actualizan con Crossref. Finalmente, los artículos son presentados con título y resumen estructurado en inglés y español en la página oficial de Acta Pediátrica de México (www.actapediatrica.org. $\mathrm{mx}$ ) en donde la interacción con el usuario es más amigable y directa.

Durante el 2016 hemos establecido un programa de capacitación técnica especializada de las herramientas computacionales disponibles, así como su personalización e incorporación en el flujo de trabajo de nuestra plataforma OJS, con el fin de que después de aprobar las pruebas de diagramación se generen las versiones finales en formatos PDF y XML, habilitando la citación cruzada y el seguimiento con CrossMark ${ }^{\circledR}$ de manera inmediata. Todo esto se traducirá en una mayor citación de los artículos publicados en Acta Pediátrica de México y a la larga en un mayor impacto científico de la revista.

Fuga de publicaciones de Acta Pediátrica de México

La fuga de publicaciones científicas valiosas es un fenómeno común en el campo de Ciencias de la Salud en Latino América. Los grupos de investigadores consolidados y cuyos trabajos tienen el mayor potencial de impacto en la generación de nuevos conocimientos o cambios de pensamiento, suelen enviar los resultados de sus investigaciones a revistas internacionales con mayor difusión y citación (i.e. mayor factor de impacto, Fl). También, aquellos grupos de investigadores en proceso de consolidación se ven influidos a enviar los resultados de sus investigaciones a las mismas revistas debido a las exigencias de los bien conocidos programas de "estímulos a la productividad científica", así como la inclusión o permanencia en el Sistema Nacional de Investigadores. En otras palabras, los mismos programas que promueven el desarrollo profesional en ciencia y tecnología, limitan el crecimiento de las revistas científicas nacionales.

La clasificación de Acta Pediátrica de México en Q4, con un índice $\mathrm{H}$ de 1 y la falta de un factor de impacto, generan una barrera para la colaboración de grupos de investigadores consolidados o en consolidación; no por esto, dejamos de hacer extensa la invitación a todos los miembros de la comunidad médica-científica a publicar con nosotros y mantener nuestro compromiso con la difusión de evidencia científica relevante en el ámbito de la pediatría.

Por otro lado, sabemos que la publicación en inglés es indispensable para lograr mayor difusión de los resultados de las investigaciones realizadas en Latino América; sin embargo, estamos convencidos que hacerlo exclusivamente en inglés limita el retorno de la información e impacto sobre la población de la cual partió el conocimiento.

Incorporación a índices, acervos bibliográficos o directorios y visibilidad internacional

Acta Pediátrica de México se ha incorporado desde 2013 como miembro a "Publishers International Linking Association, Inc (PILA)" para hacer uso del servicio/organización Crossref ${ }^{\circledR}$ permitiendo la asignación, registro y uso de identificadores digitales de objetos (DOI). También hemos obtenido el reconocimiento e incorporación al Índice de Revistas Mexicanas 
de Investigación Científica y Tecnológica del CONACyT (http://revistascientificasconacyt.unam. edu/\#/archive/1) y se ha incorporado al "Directory of Open Access Journals (DOAJ)" (https:// doaj.org/toc/2395-8235); al Índice de la Red de Revistas Científicas de América Latina y el Caribe, España y Portugal (Redalyc). (http://www.redalyc. org/revista.oa?id=4236\&tipo=colección); al Índice MedicLatina (EBSCO) (https://www. ebscohost.com/titleLists/lth-coverage.htm?_ga $=1.109857797 .1260341790 .1476394877) ; \mathrm{al}$ Emerging Sources Citation Index de Thomson Reuters (http://ip-science.thomsonreuters.com/ cgi-bin/jrnlst/jlresults.cgi); al Índice Scopus ${ }^{\circledR}$ facilitando el cálculo del Índice H (Scimago Journal \& Country Rank-SJR). (http://www. scimagojr.com/journalsearch.php?q=2110039 5059\&tip=sid\&clean=0); al índice de Google Scholar desde 2014 con índice H5 de 8 y mediana H5 de 9 (https://scholar.google.com.mx/ citations?hl=es\&view_op=search_venues\&vq= Acta+Pediatrica+de+Mexioco).

Asimismo, Acta Pediátrica de México continua su presencia en el Índice del Sistema Regional de Información en Línea para Revistas Científicas de América Latina, el Caribe, España y Portugal (latindex) con puntaje de 34/36 (http://www.latindex.org/latindex/ ficha?folio=14426) y en el Índice de Revistas Médicas Latinoamericanas (Medigraphic) (http:// new.medigraphic.com/cgi-bin/publicaciones.cgi?IDREVISTA $=74 \&$ NOMBRE $=$ Acta $\% 20$ Pedi\%E1 trica\%20de\%20M\%E9xico).

Cumplimiento con la frecuencia de publicación

Acta Pediátrica de México se ha publicado de manera ininterrumpida desde 1980. Se publica puntualmente cada dos meses en línea y en versión impresa. Nuestra publicación tiene dos páginas web: una dedicada específicamente para los procesos de gestión editorial y repositorio de nuestro archivo histórico -2010 a la fecha- (www. ojs.actapediatrica.org.mx), y otra que ofrece una interfaz amigable con el usuario en español e inglés (www.actapediatrica.org.mx) y que a su vez funge como estrategia de financiamiento de la revista (participación comercial de industria privada). Todos los artículos son descargables sin costo alguno. Ambas páginas se encuentran bajo el mismo dominio, y contamos con respaldo de la información en al menos 3 servidores distintos. En los siguientes años Acta Pediátrica de México aplicará para incorporarse a la red de resguardo de la información bajo la política LOCKSS (Lots of Copies to Keep Stuff Safe).

Para este 2017, buscará consolidarse como una de las publicaciones médicas pediátricas con un impacto real en América Latina; para conseguir este objetivo, constantemente estaremos evaluando y realizando mejoras en nuestros procesos editoriales; implementaremos estrategias de difusión en dos sentidos: por un lado, difundiendo los artículos más relevantes publicados en Acta Pediátrica de México, esto con el objetivo de visibilizar el trabajo que realizan nuestros investigadores y, por otro lado, buscaremos generar una red de grupos de investigadores consolidados o no, cuyos trabajos impacten en la generación de nuevos conocimientos o cambios de paradigmas en el ámbito de la pediatría. Es por lo anterior, que invitamos a nuestros lectores a formar parte de esta publicación y a generar juntos materiales de calidad que permitan generar y mejorar los modelos de atención a la salud durante la infancia y la adolescencia.

Dr. Francisco J. Espinosa Rosales Editor en Jefe

Dr. Armando Partida Gaytán Coeditor

M en E. Cinthya Tapia Ponce Editora Ejecutiva

Lic. Edgar Rivas Zúñiga

Corrección de estilo y asistente editorial

editor@actapediatrica.org.mx DOI: http://dx.doi.org/10.18233/APM38No2pp79-821358 